\title{
Nonresectional Simplified Folding Technique in Robotic Mitral Valve Plasty: Comparison with Leaflet Resection Technique
}

\author{
Tatsuya Tarui, MD, Norihiko Ishikawa MD, Ryuta Kiuchi MD, Shigeyuki Tomita MD, \\ Hiroshi Ohtake MD, and Go Watanabe MD \\ Department of Cardiovascular Surgery, NewHeart Watanabe Institute, Tokyo, Japan
}

\section{ABSTRACT}

Objective: The mitral valve plasty (MVP) technique for degenerative disease is typically leaflet resection and suturing. However, this technique is time consuming and unreproducible. To overcome this disadvantage, we developed a nonresectional folding technique, which is fast and reproducible. In this report, we examine our new folding technique in robotic MVP.

Methods: The new folding technique was performed in 10 patients (age $56 \pm 15$ years), and the conventional resection and suturing (RS) technique was performed in 22 patients (age $53 \pm 8$ years). In our new folding technique, we used two sutures to fold the prolapsed leaflet to the left ventricle side. The first folding suture line is a land mark, and the second line adjusts the height of the posterior leaflet to the anterior leaflet so that sufficient coaptation depth can be obtained.

Results: MVP was successful in all patients. In the folding technique group, the operation time, cardiopulmonary bypass time, and cross clamp time was faster than the conventional RS technique group $(188 \pm 31,97 \pm 32$, and $55 \pm 3 \mathrm{~min}$, versus $242 \pm 51,137 \pm 25$, and $70 \pm 15 \mathrm{~min} ; P<.05$ ). Hospital stays were significantly shorter in the folding technique group $(13 \pm 2$ days versus $17 \pm 7$ days; $P<.05)$. All patients were discharged without complications. The post-echocardiography revealed no mitral valve regurgitation in any patient.

Conclusion: The new folding technique facilitated efficient MVP for posterior leaflet prolapse in mitral valve regurgitation, without the need for the resection of the leaflet.

\section{INTRODUCTION}

Innovations in surgical approaches have enabled a minimally invasive access to the mitral valve. Recently, robotassisted mitral valve surgery has been used as an alternative, less-invasive method, and there have been many reports of successful results with robotic mitral valve plasty (MVP) [Suri 2016; Suri 2015; Ramzy 2014; Suri 2011; Chitwood 2008]. The repair technique may sometimes be same as the

Received Fanuary 1, 2017; accepted April 13, 2018.

Correspondence: Tatsuya Tarui, MD, Department of Cardiovascular Surgery, NewHeart Watanabe Institute, 3-19-11 Hamadayama, Suginami, Tokyo, Japan 168-0065; +81-3-33111119; fax: +81-3-33113119 (e-mail: tatsuya. tarui@gmail.com). conventional sternotomy; however, not all conventional techniques can be applied or are suitable for the robotic approach.

The most common treatment procedure for posterior mitral leaflet (PML) prolapse is a resection and suture (RS) technique, and in patients requiring broad PML resection, a sliding technique is used. Both of these procedures are time consuming, irreversible, and require precise leaflet cutting and suturing. Knot tying is a difficult and time-consuming procedure, especially in robot-assisted surgery. In order to simplify the plasty technique, we adopted a procedure involving folding of the prolapsed leaflet. This is a nonresectional technique that involves the inversion of the prolapsed segment

Table 1. Demographics, Comorbidity, and Valvular Pathology

\begin{tabular}{|c|c|c|c|}
\hline & Folding technique & RS technique & $P$ \\
\hline Patients & 10 & 22 & \\
\hline \multicolumn{4}{|l|}{ Demographic data } \\
\hline Male & $7(70 \%)$ & $14(63 \%)$ & .84 \\
\hline Age (years) & $56 \pm 15$ & $53 \pm 8$ & .58 \\
\hline Body mass index $(\mathrm{kg} \cdot \mathrm{m} 2)$ & $23 \pm 2$ & $23 \pm 3$ & .68 \\
\hline \multicolumn{4}{|l|}{ Comorbidity } \\
\hline Atrial fibrillation/flutter & 0 & 3 & .21 \\
\hline \multicolumn{4}{|l|}{ NYHA } \\
\hline 1 & $8(80 \%)$ & $18(82 \%)$ & .9 \\
\hline II & $2(20 \%)$ & $4(18 \%)$ & .9 \\
\hline III & 0 & 0 & - \\
\hline IV & 0 & 0 & - \\
\hline Ejection fraction & $63 \pm 7$ & $66 \pm 9$ & .49 \\
\hline \multicolumn{4}{|l|}{ Valvular pathology } \\
\hline \multicolumn{4}{|l|}{ Mitral regurgitation grade } \\
\hline $3+$ & $1(10 \%)$ & $3(14 \%)$ & .77 \\
\hline $4^{+}$ & $9(90 \%)$ & $19(86 \%)$ & .77 \\
\hline Segment P1 diseased & $1(10 \%)$ & $5(23 \%)$ & .39 \\
\hline Segment P2 diseased & $8(80 \%)$ & $11(50 \%)$ & .11 \\
\hline Segment P3 diseased & $1(10 \%)$ & $6(27 \%)$ & .27 \\
\hline
\end{tabular}

RS indicates resection and suturing; NYHA, New York Heart Association. 


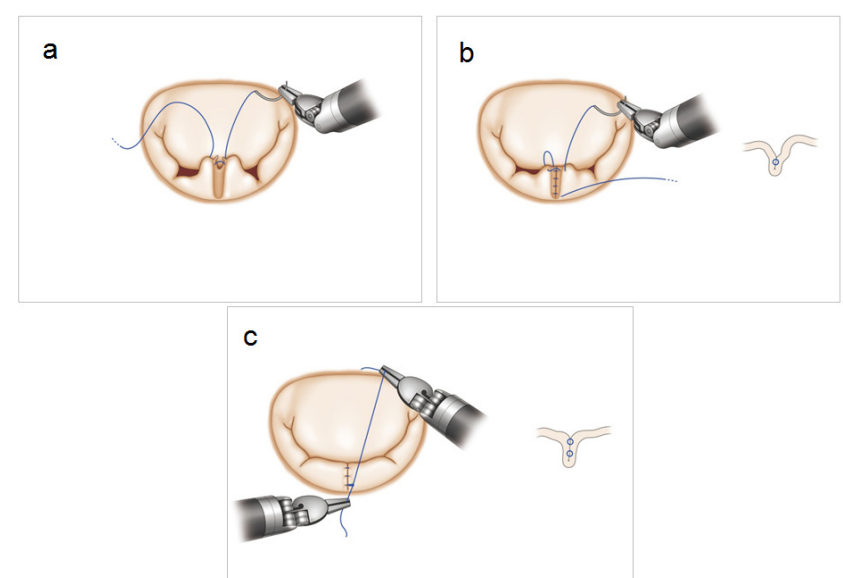

Suturing method. A, The first suture was vertically placed at the free edge of the prolapsed leaflet and tied. B, Continuous suture was placed from the leaflet toward the annulus. In the first running suture, the folding of the leaflet is kept shallow. C, The other side of the polypropylene was continuously sutured at the same suture line. In this suture, the folding is deeper, adjusting the height of the posterior leaflet to the anterior leaflet.

into the left ventricle. The present study aims to evaluate the simplicity and efficacy of this folding technique in robotic MVP and is the first report on this procedure.

\section{MATERIALS AND METHODS}

This study was approved by the Institutional Review Board of the NewHeart Watanabe Institute. Written informed consent was obtained from all the participants before starting with the study. We use the da Vinci S surgical system (Intuitive Surgical Inc, Sunnyvale, CA, USA) to perform totally endoscopic MVP via four ports.

From December 2015 to August 2016, the folding technique was used in 10 consecutive patients with severe PML prolapse. Before we adopted the folding technique, 22 patients had undergone conventional RS technique for PML prolapse. These patients were used as the control group. All patients had severe mitral regurgitation due to PML prolapse. Preoperative characteristics are summarized in Table 1.

\section{Operative Technique}

Under general anesthesia, a double lumen endotracheal intubation was used for single lung ventilation. A remote cardiopulmonary bypass was established with vacuum-assisted venous drainage from the internal jugular, right femoral vein, and common femoral artery for the arterial return. After establishing cardiac arrest using cold cardioplegia, the mitral valve was approached via a right-side left atriotomy. The prolapsed leaflet was confirmed using saline regurgitation test.

In the folding technique group, 4-0 monofilament polypropylene was placed vertically at the free edge of the prolapsed leaflet and tied (Figure a). After knotting, a continuous suture was placed, extending from the leaflet toward the
Table 2. Operative Data

\begin{tabular}{lccc}
\hline & Folding technique & RS technique & $P$ \\
\hline Patients & 10 & 22 & - \\
Procedure & & & \\
mitral valve plasty & 10 & 22 & - \\
mitral valve replacement & 0 & 0 & - \\
Peri-operative data & & & \\
Operation time & $188 \pm 31$ & $242 \pm 51$ & $<.05$ \\
Cardiopulmonary bypass time & $97 \pm 32$ & $137 \pm 25$ & $<.05$ \\
Aortic cross clamp time & $55 \pm 3$ & $70 \pm 15$ & $<.05$ \\
Transfusion (\%) & $2(20 \%)$ & $4(18 \%)$ & .9 \\
Post-operative data & & & \\
ICU stay & $1.1 \pm 0.3$ & $1.3 \pm 1.0$ & .39 \\
hospital stay & $13 \pm 2$ & $17 \pm 7$ & $<.05$ \\
mortality & 0 & 0 & - \\
Ejection fraction & $62 \pm 2$ & $64 \pm 3$ & .45 \\
Atrial fibrillation & 1 & 3 & .77 \\
Residual mitral valve regurgitation & & 11 & .6 \\
none & 6 & 0 & .49 \\
trivial & 4 & 10 & - \\
mild & 0 & 0 & - \\
moderate & & & \\
severe & & & \\
\hline
\end{tabular}

ICU indicates intensive care unit.

annulus (Figure b). In the first running suture, the folding of the leaflet was kept shallow. Thereafter, the other side of the polypropylene was continuously sutured at the same suture line to create the second running suture (Figure c). In this suture, the folding was deeper; the height of the posterior leaflet was adjusted to the anterior leaflet so that sufficient coaptation depth could be obtained. The schema is presented in Figure and the supplemental video. In the conventional RS technique group, the prolapsed leaflet was quadrangularly resected with a scissor arm, and sutured with interrupted sutures.

After the leaflet plasty procedure, a Carbomedics Annuloflex ring (Sorin, Arvada, CO, USA) was sutured to the valve annulus with continuous suturing, using the V-Loc barbed suture (Medtronic, Minneapolis, MN, USA). This technique has been previously presented by Watanabe et al [Watanabe 2015].

\section{Statistical Analysis}

Continuous variables are expressed as mean \pm standard deviation. Student's t-test and Pearson's $\chi^{2}$ tests were used to analyze continuous and categorical variables, respectively. Results were considered significant at $P<.05$. Statistical analyses were done with JMP version 12 (SAS Institute). 


\section{RESULTS}

Operative and postoperative data are presented in Table 2. All the patients successfully underwent repair with both techniques. There were no mitral valve replacements. Most patients had isolated P2 PML prolapse. Outcomes are presented in Table 2. The operation time, cardiopulmonary bypass time, and aortic cross-clamp time were $188 \pm 31,97$ \pm 32 , and $55 \pm 3 \mathrm{~min}$, respectively, for the folding technique and $242 \pm 51,137 \pm 25$, and $70 \pm 15 \mathrm{~min}$, respectively, for the RS technique group. All of the aforementioned times were significantly shortened using the folding technique $(P<.05)$. No patient had systolic anterior motion of the mitral valve. There was no significant difference in the intensive care unit (ICU) stays (folding technique versus RS technique: $1.1 \pm$ 0.3 days versus $1.3 \pm 1.0$ days; $P=.39$ ), however, the hospital stays were significantly shorter $(13 \pm 2$ days versus $17 \pm 7$ days; $P<.05)$. Follow-up transthoracic echocardiography revealed trivial or less residual mitral valve regurgitation, though one patient revealed mild regurgitation in RS technique group. All patients were discharged without complications, such as operative death or strokes.

\section{DISCUSSION}

In the present report, the folding technique for PML prolapse has been shown to be a simple and effective technique compared to the conventional RS technique for robotic MVP.

Resection and annuloplasty has been the most common technique for degenerative posterior leaflet prolapse; however, a significant disadvantage of this method is the irreversibility [Carpentier 1983]. If the resection is too large and regurgitation persists, the additional repair may be difficult and be converted to replacement. Conversion to replacement is difficult, particularly while using the robot-assisted totally endoscopic approach, because the port is smaller than the replacement valve. This size difference makes it difficult to insert the valve without conversion to small thoracotomy.

The folding technique has several advantages: it is fast, simple, and reproducible, enabling repeated repairs to obtain desired results. In the present report, we were able to shorten the operation, cardiopulmonary bypass, and aortic crossclamp times compared to RST. Because of the reproducibility, we were able to obtain satisfactory MVP, and no mitral valve regurgitation was found in any of the patients in the postoperative echocardiography.

Methods for nonresectional MVP have been reported previously. Tabata and colleagues, and Mihaljevic and colleagues, reported horizontal folding valvuloplasty without leaflet resection [Tabata 2008; Mihaljevic 2006]. Tsukui and colleagues reported a vertical folding plasty with good post-operative results [Tsukui 2013]. However, there have not been any reports involving the use of an endoscopic or robotic approach. All of the previous reports for the folding technique were performed with interrupted sutures. To apply the folding technique to an endoscopic or robotic approach, we used continuous sutures, which is an effective and practical method. The first continuous suture can be a landmark for the suture line, and the second can adjust coaptation height to the anterior leaflet and secure the folded leaflet. When compared with the use of a single suture, the repaired leaflet is stronger when using two suture lines, preventing leaflet tissue cutting. Furthermore, the folding technique requires knot tying only twice, which enables the MVP to be fast and simple.

Posterior leaflet prolapse and single segment prolapse are indications for folding plasty. We did not apply this technique to anterior leaflet prolapse or large segment prolapse. Tsukui and colleagues have reported successful MVP to all and multiple segment prolapse [Tsukui 2013]. Further studies are needed to determine whether this folding technique is applicable in an endoscopic or robotic approach. Furthermore, this study was retrospective, and reported only early outcomes. A randomized and long-term follow-up study is necessary to assess the efficacy and durability of this technique.

We believe that this technique will be useful and efficacious in not only the robotic, but also the conventional approach, though long-term follow-up of the patients is essential.

\section{REFERENCES}

Carpentier A. Cardiac valve surgery-the "French correction." 1983. J Thorac Cardiovasc Surg 86:323-37.

Chitwood WR, Rodriguez E, Chu MWA, et al. 2008. Robotic mitral valve repairs in 300 patients: a single-center experience. J Thorac Cardiovasc Surg 136:436-41.

Mihaljevic T, Blackstone EH, Lytle BW. 2006. Folding valvuloplasty without leaflet resection: simplified method for mitral valve repair. Ann Thorac Surg 82: e46-8.

Ramzy D, Trento A, Cheng W, et al. 2014. Three hundred roboticassisted mitral valve repairs: the Cedars-Sinai experience. J Thorac Cardiovasc Surg 147:228-35.

Suri RM, Burkhart HM, Daly RC, et al. 2011. Robotic mitral valve repair for all prolapse subsets using techniques identical to open valvuloplasty: establishing the benchmark against which percutaneous interventions should be judged. J Thorac Cardiovasc Surg 142:970-9.

Suri RM, Dearani JA, Mihaljevic T, et al. 2016. Mitral valve repair using robotic technology: safe, effective, and durable. J Thorac Cardiovasc Surg 151:1450-4.

Suri RM, Taggarse A, Burkhart HM, et al. 2015. Robotic mitral valve repair for simple and complex degenerative disease: midterm clinical and echocardiographic quality outcomes. Circulation 132:1961-8.

Tabata M, Ghanta RK, Shekar PS, et al. 2008. Early and midterm outcomes of folding valvuloplasty without leaflet resection for myxomatous mitral valve disease. Ann Thorac Surg 86:1388-90.

Tsukui H, Umehara N, Saito H, et al. 2013. Early outcome of folding mitral valve repair technique without resection for mitral valve prolapse in 60 patients. J Thorac Cardiovasc Surg 145:104-8.

Watanabe G, Ishikawa N. 2015. Use of barbed suture in robot-assisted mitral valvuloplasty. Ann Thorac Surg 99:343-5. 